\title{
Integrative interpretation of QI and Body sensations
}

\section{Editorial}

Body sensations are of great importance in the field of integrative medicine, both in diagnosis and therapy. With their help, we can determine the location of "absence" or "stagnation," and we can make treatment more effective by paying attention to the sensations. From a superficial point of view, it may seem that bodily sensations are primarily related to where we feel them. Classical Eastern medical doctrines also build on this, the description of which can be found in the theories of qi (prana) deficiency and qi stagnation. However, the results of modern science are increasingly guiding us not to take the signals of our bodies for granted.

\section{Sources of body sensations}

Body sensations have subjectivity. One of their components is tied to the specific place where the feeling develops. Their other component is related to the functioning of the nervous system and the brain, which amplify, weaken, or transform the sensation. If we feel pain somewhere in our body, the interesting way is not the local influences that are definitely present, in fact, not even the activity of the sensory nerve coming from there, but the perceptual function of our brain. For example, phantom pain cannot be a local factor, as the pain occurs in a non-existent (e.g., amputated) part of the body. And if, during hypnosis, we tell the patient to burn his skin but touch it with just a pen, the brain colors this stimulus into burning pain. The most direct stimulus can be triggered during wakeful brain surgeries - simply by stimulating the appropriate areas of the brain, bodily pain can be caused. But even in a modified state of consciousness, we may be able to generate a sense of physical pain through our purely conscious activity.

However, unless local perception is absolutely necessary for the sensation to develop, we cannot be sure that a pain in the body, the origin of which we cannot pinpoint, is actually from that place, possibly from damage to the sensory nerve (e.g. sciatica), or "only" is projected there by our brain. The same question arises in other body sensations, especially the so-called at a "qi" feeling. Obviously, if we feel qi during an acupuncture needle treatment, the reason is the sting - even if we feel it at a different point than where the needle penetrated under the skin. But what about other spontaneous qi sensations? And especially with those we create for ourselves through meditation, concentration, and nei (inner) qi-gong practice? How do we know that we have actually initiated a bodily change and feel it, or have we created a feeling in our brain that is projected onto a place in that body? Actually out of nowhere. And although the most common specific stimulus for body sensations and pain is its origin, it is always modified by our nervous system according to our current state of consciousness and metabolism. Moreover, the more attention we pay to the observation of bodily sensations, the more we will be able to create them by our minds as well. Biofeedback research provides a good example of this. The difficulty arises where this phenomenon can occur not only consciously but also subconsciously. This is the process of projection. In this case, we are unaware of the origin of the feeling, and perhaps by perceiving and awareness of it, we look for local factors in the background - even if they are not present. In the wrong case, we label it a symptom, we start to worry, we focus our attention on it, and with that we intensify it even more. This
Volume 14 Issue 4 - 202I

\author{
Károly Joós, Hegyi Gabriella \\ Department of Integrative medicine, Pecs University, Hungary
}

Correspondence: Joós Károly, Department of Integrative medicine, Pecs University, Hungary, Email drhegyi@hu.inter.net

Received: July 01, 202I | Published: July 08, 2021

mechanism is often seen in action in psychogenic pain syndrome. However, we tend to produce theories about the origins of feelings, and with these we can easily mislead ourselves or our doctors. In the old days, when Eastern medical systems were created, people didn't even know about nerve cells, electrons, or hormones and the immune system. Although the functioning of consciousness has been studied a lot, as psychic processes are difficult to measure and quantify, their subjective nature, as it is today, has greatly hampered the scientific approach. The ancient physicians thus relied on their observations and experiences, and summed up everything they found about the functioning of the organization. To describe the function, the concept of qi or prana was introduced, which covered physiological processes based on both electrical phenomena and the chemical pathway, as well as psychic functioning. According to the esoteric approach, there is also an immeasurable, invisible energetic phenomenon, which is parapsychology, resp. belongs to the subject of metaphysics. And while scientific research has failed to prove the reality of this, they cannot rule out the possibility. Thus, this area is like faith in God: everyone's own worldview determines what they believe in. However, science is necessarily a reductionist, dealing only with the phenomena it can study - it cannot interpret the world of esotericism and metaphysics accordingly. In this writing, the goal is to strengthen connection and communication with the world of science, so I will not deal in detail with esoteric explanations. Moreover, wherever possible, I offer alternative explanations for the interpretation of seemingly supernatural phenomena based on physiological processes and psychological phenomena. But it is important to note that this is only a valid explanation according to a particular worldview, it cannot be ruled out that it is incomplete accordingly.

\section{Qi and qi sensation}

The concept of qi is worth knowing that it is used quite flexibly in Eastern teachings. In some ways, all matter and energy, that is, the whole of the Universe, is made up of qi, its various subtle forms. Spectrum is usually described by the yin-yang pair, so energy is the yang-like form of qi, while matter is yin-like. The processes of the human consciousness are formed by yang-type qi, and the tangible manifestations of the human body are formed by yin-type qi. If we further narrow the definition, the most yang phenomena (soul, psyche, consciousness) are defined as shen, yin phenomena as fluids or organs, and the boundary between the two as qi. And even this can be further divided e.g. qi forms represented by genetic information, the energy content of nutrients, or the heat of our bodies. Henceforth, however, qi, which we define as bodily sensation, is a rather narrow concept, 
which, moreover, is described in slightly different ways in the various classic books. So, ultimately, we have a great deal of freedom in how we use the concept of qi, so we can name many physiological but even psychic phenomena. Although various teachers and classical schools often give the impression that qi is a well-defined and well-defined concept, this is not the case at all. It is enough to ask the different masters and look more closely at the teachings of each school - we will find significant differences. Thus, qi as a bodily sensation can result either from local bodily processes or from the projection of central stimuli of the nervous system, and it is sometimes very difficult to distinguish between the two. For example, during acupuncture treatment, sympathetic nervous system reactions occur when a needle is inserted, which may include increased circulation, possibly tingling, opening of the chest, shoulders, nasal cavity, and thus easier breathing, these are local effects. Later, at the time of retaining or gently manipulating the needles, parasympathetic nervous system effects dominate, the patient relaxes, bowel movements begin, and urine excretion increases - which can be observed for days. These are also local effects that can become more and more permanent by modulating the equilibrium state of the autonomic nervous system. In addition, however, the doctor asks his patient to monitor physical sensations. This means focusing on the small, even subtle feelings that are already present, which magnifies the feelings in the treated area - centrally. Moreover, even consciousness as a whole can create a sensation away from the site of needle punctures, especially if the doctor predicts their occurrence.

\section{Diagnostics based on special skills and intuitions}

Another phenomenon can be observed when the doctor "feels the qi" in the patient's body. Is it possible that we can tune in to another person so much that we can feel the tiny vibrations of his body and diagnose his condition using "subtle perception"? Maybe so - although there is currently no serious scientific evidence for this. However, another possibility emerges if we scan the functioning of our brain or mind. This could not be studied in ancient Eastern cultures due to a lack of adequate knowledge and tools, so they sought an explanation without neurons and electrical impulses. There is no problem with that. However, today, when we have a vast body of scientific knowledge compared to antiquity, it is worth reinterpreting this issue. So how can we describe the qi perceived in the bodies of others in the language of the sciences? What is the logical explanation for the fact that many doctors, whether thousands of years ago or today, base their diagnoses on what they "see" in a patient's body with their subtle senses? If we examine this phenomenon, we find that it is not an easy skill to acquire; it takes years, even decades to practice to develop (more or less) accurate diagnostic skills. During all these years, however, the doctor's traditional diagnostic skills and, on that basis, his intuitions inevitably develop. As soon as the patient enters, moves, speaks, such as his or her shape, face, tongue, etc., he or she forms a very good picture of what is going on in his or her client's body. To this comes practical experience, especially when working with body treatments. If I massage here, it happens, if I stab it there, then it is. I can achieve this with a laser, cupping it, and a joint manipulation technique. This confirms and refines diagnostic skills, while over the years; the physician will become more and more aware of not only the patient's illness, but also its pattern of manifestation affecting the whole body. However, much of this intuition is not the result of rational thinking. And although it may stem from the material knowledge and experience accumulated over the years, decades, the professional will not necessarily be aware of this. How could you get out of your many internal sources exactly where your intuitions come from? And so, as intuitions become more reliable over the years, their source becomes increasingly obscure. We may even think that our intuitions come through "subtle perception." Because we walk next to the patient, put our hands over it and feel it. We look at him in a modified state of consciousness and see. Of course, that should be the case, how could it be otherwise? ... and yet it can be. After all, projection works here as well. If I have an intuition about the diagnosis, the patient's pattern of functioning, it is easily projected onto the patient's body by my brain, and I will be able to feel that my own projection. And if others look at the same patient, colleagues who have more or less similar knowledge and experience, they will be able to feel it too. A little different for everyone, but in terms of the underlying factors (where the biggest deficit or stagnation is) is similar. And this can give the false impression that subtle perception does exist, because there are many of us here, well-trained and wellseen, learned colleagues, and we all feel the same way. However, this is not necessarily the case. We always project our own subjective worldview onto objective reality - necessarily.

To this, we could say that the effectiveness of treatment based on the diagnosis of subtle perception will prove that the theories behind the method are indeed correct. But, as follows from the above, this is not true. After all, the diagnosis itself is correct, only its source is different. The intuition that comes from my knowledge and experience is often surprisingly accurate. Even if I am aware that I have acquired this intuition in a special way, the treatment put together based on it will work. That's exactly why I worked and studied for decades.

\section{Remote diagnosis and treatment?}

The following topic is given by the cases where remote diagnosis and / or remote treatment takes place. This method still exists to this day, and is practiced by many, not only by fast-paced eso-shamans, but also by highly trained medical naturopaths. And this is based on practical experience that these methods can also work. It may seem as if the phenomenon of remote diagnosis justifies the reality of special, elusive energies, but this is deceptive. In this case, two traditional factors may explain the effectiveness of the therapy. One is that many times you get so much information (complaints, description of symptoms, medical history, other diagnostic signs) that your intuition can work well, quite accurately, and the treatment will be good. The other is precisely the peculiarity of the journey, and our (hidden) desire for supernatural phenomena to be real. If a doctor believes in a method and trusts his or her colleague with long-term help, he or she will have strong confidence in the intervention he or she is proposing. On the other hand, due to his uncertainty, he could not feed so much faith into the protocol compiled on the basis of his own intuitions and knowledge. If you resolutely, come to your patient this time with the good inner feeling that you now have the best treatment, the best way, this faith gives him and the treated person strength, new impetus, and stronger faith than before. Together, they are indeed able to provide more effective treatment, this time in accordance with the laws of psychology and psychosomatics. Telemedicine can be an excellent example of the power of the placebo effect. In most of these practices, the therapist determines the time of treatment and instructs the patient to rest, relax, and monitor body sensations. And in doing so, the effects of expectations, desire to meet, relaxation, and focus on body sensations add up, triggering truly measurable physiological changes in the body. Under optimal conditions, this treatment can awaken such a strong belief in the patient that even a real healing process can begin in his body. This, in turn, can be verified in retrospect by medical research tools. Thus, the placebo effect optimally not only causes temporary improvement, but can also be the basis for healing. 


\section{Conclusions}

However, based on the above, there is no need and no judgment to be made about healing work based on the perception and control of qi and body sensations. We are not in possession of certain knowledge. At present, we cannot know or prove whether there are subtle perceptions, energies that can be caught up in healing work. In ancient times, the most probable explanation was accepted as fact, describing qi and the possibilities of perceiving and controlling it. Until a better explanation was found, they worked with it, and many still work to this day. However, while subtle perceptions and energies have not been scientifically substantiated so far, there are other possibilities that can satisfactorily explain the same phenomena. To do this, one must look for answers beyond physiological knowledge, in the realm of psychology and psychosomatics. Accordingly, we will have two possible explanations for special feelings and cures. One is a narrative based on thousands of years of tradition, but now much attacked, often refuted, and not scientifically justified. The other, on the other hand, rests on scientifically known and recognized foundations; its logic meets modern expectations, and is also able to fully explain the phenomena. We can all choose which one to accept. And we can do this without a value judgment, because regardless of the explanations, the method works, our conscience can remain clear anyway. On the practical side, however, it is important to address another question: what is the direction of development, where is the medicine going? For at present, it seems that due to the immeasurably complex functioning of the human body and the astonishingly complex pathways of disease, we will need the insights of experienced, muchseen, learned colleagues for a long time to come. In both diagnostics and therapy - integrative medicine provides an excellent field for this.
We can benefit a lot from correctly interpreting tiny bodily signs, feelings, states of consciousness, and psychosomatic processes during diagnosis. This makes it easier to determine pathogens, to deal with physical, chemical, or mental processes, and to make a more accurate therapeutic plan. However, a language that is understood by as many colleagues and health professionals as possible is more practical. It used to be the language of qi, yin-yang, and body fluids; nowadays it is more of medicine and psychosomatics. There may be doctors and other professionals who are doing better with the former, but in the long run, the opportunity to collaborate and move forward together may prove more valuable. And the direction for this seems clear in terms of a "common language" that everyone can understand. Be generally interpretable and accepted, so use modern, science-based concepts. Exactly the same is true during therapy. If we integrate methods that affect the body and the soul, we get better results. And for a psychologist, a doctor, a lifestyle therapist, and a physiotherapist to understand each other well, to work together in the long run, a common language is also essential. And from the fact that our mother tongue is something, we can learn another one.

\section{Acknowledgments}

None.

\section{Conflicts of interest}

Author declares there are no conflicts of interest.

\section{Funding}

None. 